\title{
An unusual cause of heart failure in postoperative Tetralogy of Fallot
}

\author{
GURBHEJ SINGH ${ }^{1}$, Arun Gopalakrishnan ${ }^{2}$, and Sivasankaran $\mathrm{S}^{2}$ \\ ${ }^{1}$ Hero DMC Heart Institute \\ ${ }^{2}$ Sree Chitra Tirunal Institute for Medical Sciences and Technology
}

May 6, 2020

\begin{abstract}
The long term results of Tetralogy of Fallot (TOF) repair have been good, pulmonary regurgitation and consequent right ventricular dilatation is the most common long term sequel of definitive surgery. Even in such a setting, overt systemic venous congestion is limited to the rare setting where right ventricular dysfunction sets in due to deferred surgery or progressive arrhythmia. Here we report a unique case where a patient developed right heart failure from an unexpected etiology 14 years after TOF repair.

\section{Introduction:}

The long term results of Tetralogy of Fallot (TOF) repair have been good, pulmonary regurgitation and consequent right ventricular dilatation is the most common long term sequel of definitive surgery. Even in such a setting, overt systemic venous congestion is limited to the rare setting where right ventricular dysfunction sets in due to deferred surgery or progressive arrhythmia. Here we report a unique case where a patient developed right heart failure from an unexpected etiology 14 years after TOF repair.
\end{abstract}

\section{Case Report:}

A 14-years-old boy born of non-consanguineous parents, hailing from Kanyakumari, along the southern coast of India, was diagnosed with TOF and referred to our institute. He underwent a cardiac catheterization and was found to have elevated right-sided filling pressures and systemic right ventricular (RV) pressures. The right atrial mean pressure was $15 \mathrm{mmHg}$ and right ventricular end-diastolic pressure was $26 \mathrm{mmHg}$. He underwent trans-atrial intracardiac repair with a transannular patch. The postoperative period was complicated by right-sided pleural effusion which was managed with intercostal drain for 6 days. On follow up he was noted to have a tiny residual perimembranous ventricular septal defect and was kept on conservative management. He was lost to follow up five years after surgery.

He presented again at 40 years of life with functional class II dyspnea on exertion for 6 months and recentonset pedal edema. He was noted to be in atrial fibrillation with controlled ventricular rate. The mean jugular venous pressure was elevated with prominent $\mathrm{v}$ waves and sharp y descent. Echocardiography revealed moderate tricuspid and pulmonary regurgitation and dilated right ventricle. A restrictive perimembranous ventricular septal defect was noted shunting left-to-right during systole and right-to-left during diastole (Figure 1, A-B) . A detailed evaluation showed apical obliteration of the RV apex and reduced trabeculations. Cardiac MRI showed RV apex obliteration(Figure 1, C-D) with late gadolinium enhancement of RV apical endomyocardial. RV volumes were calculated to be $145 \mathrm{ml} / \mathrm{m}^{2}$ in end-diastole and $85 \mathrm{ml} / \mathrm{m}^{2}$ in end-systole.

After stabilization, cardiac catheterization was done to study the hemodynamics. Right atrial mean was elevated (v $28 \mathrm{mmHg}, \mathrm{m} 16 \mathrm{mmHg}$ ). There was no gradient from RV inflow to RV outflow, RV inflow (systolic 
$29 \mathrm{mmHg}$, dip diastolic $12 \mathrm{mmHg}$, end-diastolic $18 \mathrm{mmHg}$ ), and RV outflow (Systolic $28 \mathrm{mmHg}$, end-diastolic $17 \mathrm{mmHg}$ ) (figure 2) . Pulmonary artery pressures were systolic $22 \mathrm{mmHg}$, diastolic $12 \mathrm{mmHg}$ and mean $16 \mathrm{mmHg}$. There was no right ventricular outflow tract obstruction. There was an $8 \%$ step-up in oxygen saturation at the ventricular level with a shunt fraction of 1.25:1. RV angiogram showed RV apical obliteration and moderate tricuspid regurgitation. A left ventricular angiogram showed normal left ventricular contour with good ventricular function and a small sub-aortic ventricular septal defect shunting left-to-right.

He was kept on close medical management with a diuretic. He is planned for a pulmonary valve replacement with bidirectional Glenn shunt in case of recurrent heart failure or symptomatic worsening.

Discussion:

Endomyocardial fibrosis (EMF) is a dwindling disease worldwide now limited to coastal India and parts of Africa and South America ${ }^{1}$. It is characterized by fibrotic replacement of ventricular myocardium leading to impaired relaxation thus causing elevated diastolic pressures, and also valvular regurgitation. Management of EMF consists of control of heart failure and the surgical option of endocardial decortication and if required valvar repair/replacement. Further, the role of bidirectional Glenn has been reported in the advanced course of the disease ${ }^{2}$.

Ours is a unique case where this tropical disease process complicated the long term management of TOF. The index patient was documented to have elevated right-sided filling pressures at the age of 14 years. However, in the absence of ventricular dilatation or effacement, endomyocardial fibrosis was not suspected and elevated right ventricular filling pressures were attributed to the restrictive pattern seen in delayed presentation of $\mathrm{TOF}^{3}$. While a diagnosis of EMF in its inflammatory stages would have helped us initiate anti-inflammatory therapy, it seems unlikely that it would have altered the surgical plan. Since the patient tolerated the postoperative period without low cardiac output syndrome, it is clear that the right ventricular volumes were adequate for biventricular repair. Advanced stages of this disease process have been reported earlier as the development of Fontan physiology in the case of endomyocardial fibrosis ${ }^{4}$. Since the patient showed symptomatic improvement with medications, he was kept on close medical management temporarily.

The diastolic right-to-left shunt across the residual VSD was attributed to the elevation of RV end-diastolic pressures more than left ventricular diastolic pressures.

\section{Conclusions:}

In tropical countries, endomyocardial fibrosis (EMF) can present with heart failure in operated congenital heart disease including TOF. EMF can be an underlying cause for restrictive physiology of the right ventricle in delayed presentation of TOF.

\section{References:}

1. Vijayaraghavan G, Sivasankaran S. Tropical endomyocardial fibrosis in India: A vanishing disease! Indian Journal of Medical Research. 2012 Nov 1;136(5):729.

2. Mishra A, Krishna Manohar SR, Sankar Kumar R, Valiathan MS. Bidirectional Glenn shunt for right ventricular endomyocardial fibrosis. Asian Cardiovasc Thorac Ann. 2002 Dec;10(4):351-3.

3. Benbrik N, Romefort B, Le Gloan L, Warin K, Hauet Q, Guerin P, et al. Late repair of tetralogy of Fallot during childhood in patients from developing countries. Eur J Cardiothorac Surg. 2015 Mar 1;47(3):e113-7.

4. Narayan GS, Sivasubramonian S. Spontaneous Fontan physiology in burnt-out endomyocardial fibrosis. Circulation. 2012 Jan 31;125(4):e296-297.

Figure legends:

Figure 1: 
1. Continuous-wave Doppler across ventricular septal defect showing left-to-right shunt (red arrow) during systole and right-to-left shunt during diastole (yellow arrow) reflecting higher right ventricular diastolic pressure compared to the left ventricle

2. Colour M-Mode shows the left-to-right shunt (red arrow) during systole and right-to-left shunt during diastole (yellow arrow)

3. Magnetic resonance image showing RV apical obliteration (Blue arrow).

4. Magnetic resonance image showing late gadolinium enhancement of RV apex endomyocardium (Blue arrow).

\section{Figure 2:}

1. RV angiogram (RAO 30 degrees) during diastole shows apical obliteration and diverticuli resembling a caterpillar

2. RV angio during systole shows disappearances of diverticuli

3. LV angio in RAO view shows no involvement of left ventricle and site of VSD jet (red arrow)

4. Simultaneous left ventricular and right ventricular pressure traces showing RV ed $17 \mathrm{mmHg}$, LV ed 10 $\mathrm{mmHg}$. LV ed - Left ventricular end-diastolic pressure, RV ed -Right ventricle end-diastolic pressure

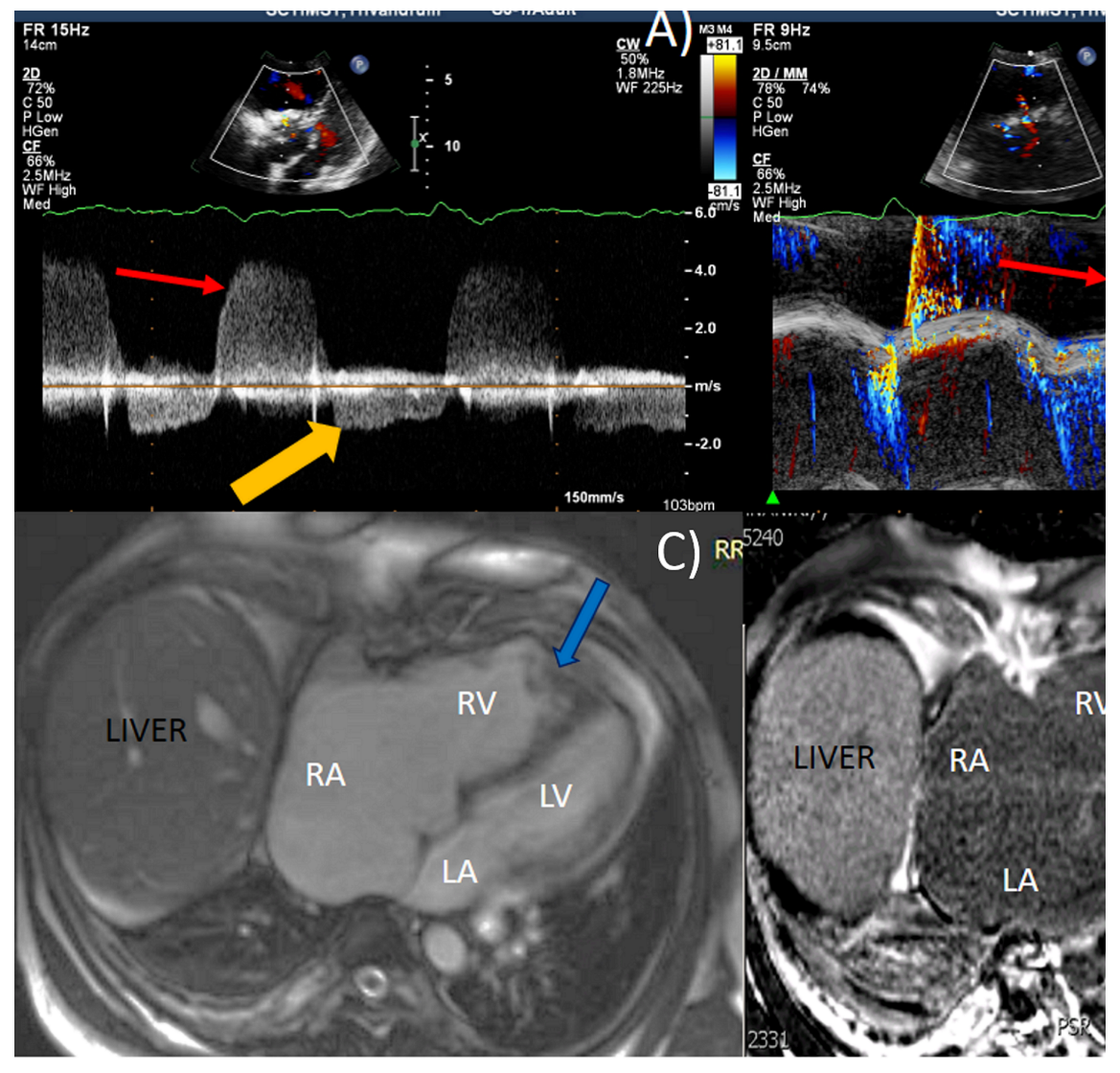




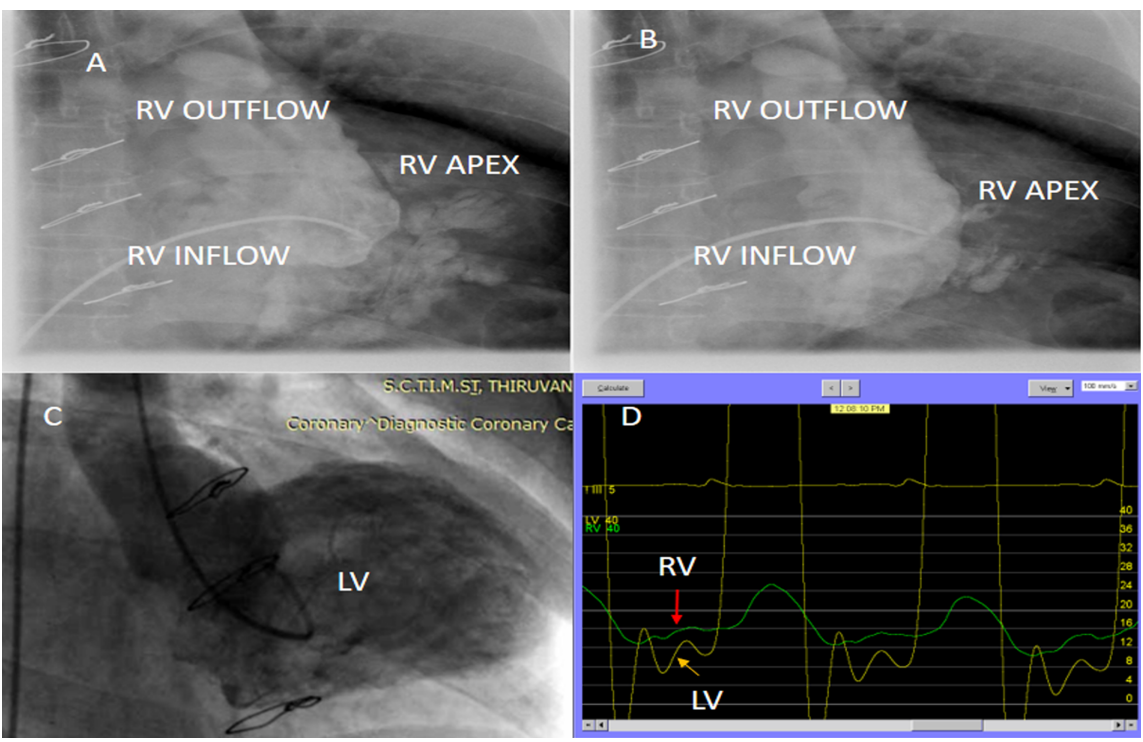

